\title{
Breed predisposition to canine gastric carcinoma - a study based on the Norwegian canine cancer register
}

Tonje Seim-Wikse ${ }^{1,3 *}$, Einar Jörundsson ${ }^{2}$, Ane Nødtvedt ${ }^{3}$, Tom Grotmol ${ }^{3,4}$, Charlotte R Bjornvad ${ }^{1}$, Annemarie T Kristensen ${ }^{1}$ and Ellen Skancke ${ }^{3}$

\begin{abstract}
Background: Previous research has indicated a breed predisposition to gastric carcinoma in dogs. However, results to date are inconsistent since several studies have failed to prove such a predisposition. Better knowledge of breeds at risk could facilitate early detection of gastric carcinoma in dogs. The aim of the study was to retrospectively investigate the proportion and possible breed predisposition to canine gastric carcinoma using the Norwegian Canine Cancer Register for calculations of proportional morbidity ratios (PMRs) for the period 1998-2009.

Results: Histologically verified tumours recorded in the Norwegian Canine Cancer Register were studied ( $n=19,715)$. A total of $31(0.16 \%)$ cases of canine gastric carcinomas were identified. The median age of affected dogs was 10 years. The most commonly reported clinical signs were vomiting, anorexia, and weight loss. Males had significantly higher odds of gastric carcinoma than females $(P=0.02)$. The PMR with 95\% confidence interval $(\mathrm{Cl})$ was calculated for each breed, and a breed predisposition was identified. Individuals of the breeds Tervuren (PMR 56.1), Bouvier des Flandres (PMR 36.5), Groenendael (PMR 34.5), Collie (PMR 26.1), Standard poodle (PMR 7.6), and Norwegian elkhound (PMR 6.1) had a significantly increased risk of developing gastric carcinoma.
\end{abstract}

Discussion and conclusion: The proportion of cases of gastric carcinoma recorded in the Norwegian Canine Cancer Register was found to be $0.16 \%$, and a breed predisposition was identified. The breed predisposition observed in the current study indicates a genetic susceptibility to gastric carcinoma.

Keywords: Dog, Gastric carcinoma, Breed, Proportional morbidity ratio, Cancer registration

\section{Background}

Canine gastric cancer is rarely diagnosed and is reported to account for approximately $0.1-0.5 \%$ of canine neoplasias [1-3]. However, it is still more prevalent in dogs than in other domestic animals [4]. The majority of gastric malignancies in dogs are carcinomas, accounting for $50-90 \%$ $[2,5,6]$, followed by leiomyosarcomas and malignant lymphoma [2]. Adenocarcinoma is the most common type of gastric carcinoma [2]. This subtype of carcinoma forms tubular structures and may exhibit various patterns at

\footnotetext{
* Correspondence: Tonje.Seim@nvh.no

'Department of Veterinary Clinical and Animal Sciences, Faculty of Health and Medical Sciences, University of Copenhagen, Frederiksberg, Copenhagen, Denmark

${ }^{3}$ Department of Companion Animal Clinical Sciences, Norwegian School of Veterinary Science, Oslo, Norway

Full list of author information is available at the end of the article
}

different levels of the invasion of the stomach wall. Undifferentiated carcinomas, which comprise the remaining carcinomas, have no visible glandular structures [4]. The clinical relevance of these histopathological subtypes of carcinoma is unknown, and the general term gastric carcinoma' is used in this article. Among dogs, gastric carcinoma has been reported to affect males more frequently than females [2,3,6-9]. A higher incidence among males is also seen for human gastric carcinoma [10]. Gastric carcinoma is generally a disease of older dogs, but has been reported in dogs in the age range $3-20$ years, with a mean age at diagnosis of $8.4-10.8$ years [2,3,5,7-9,11,12]. The incidence of gastric carcinoma increases with age also in humans [10]. Gastric carcinoma in dogs is most often located in the lesser curvature and the pyloric region of the stomach $[2,3,5,8]$.

\section{Biomed Central}

(c) 2013 Seim-Wikse et al.; licensee BioMed Central Ltd. This is an Open Access article distributed under the terms of the Creative Commons Attribution License (http://creativecommons.org/licenses/by/2.0), which permits unrestricted use, distribution, and reproduction in any medium, provided the original work is properly cited. 
The most common clinical signs associated with gastric carcinoma are vomiting, anorexia, and weight loss. Haematemesis, melaena, anaemia, lethargy, ptyalism, polydipsia, abdominal distension, and abdominal discomfort are other reported signs $[3,5,6,8,9]$. The clinical signs resemble those associated with chronic gastritis [13]. The majority of canine patients have had clinical signs for approximately four months or less by the time of diagnosis, although as much as 18 months' duration is reported $[3,5,6,8,14]$. The prognosis in cases of gastric carcinoma is poor. One case series reported a median survival time of 35 days, with a range of 0 days to 10 months after diagnosis [5]. Furthermore, 70-90\% of gastric carcinomas have metastasized by the time of diagnosis or euthanasia [3,5,7]. The most common sites reported for metastases are the regional lymph nodes, with others being the omentum, duodenum, liver, pancreas, spleen, oesophagus, adrenal glands, and lungs $[3,5,7,9]$.

A preliminary diagnosis of canine gastric carcinoma is usually obtained by ultrasound or endoscopic examination of the stomach. Using ultrasound, thickening of the gastric wall, loss of gastric wall layering, and enlargement of regional lymph nodes is likely to be observed [15]. Endoscopy allows visualization of the mucosa and performing biopsies in order to enable a definite diagnosis. A distinct ulcer with thick and irregular walls elevated from the surroundings is commonly seen, but more diffuse changes with loss of rugal folds and submucosal vascular pattern in the absence of ulcers are also described $[3,8]$.

In humans, $95 \%$ of gastric cancers are sporadic cases, and Helicobacter pylori infection is the most important environmental risk factor [16]. Familial aggregation in a small proportion of cases suggests a significant genetic predisposition $[17,18]$. Hereditary diffuse gastric cancer in humans, where the causative germ line mutation has been identified as $C D H 1$, is reported to be associated with $5 \%$ of all gastric cancer [17].

Canine breed predisposition has been reported for some types of cancer [1]. Although breed predisposition to gastric carcinoma has been suspected, several studies have failed to provide supporting evidence $[2,5,9]$. Table 1 provides a summary of predisposed breeds reported from other studies, the majority of which are case series. These case series are purely descriptive studies, with no comparison between the characteristics of subgroups. Due to this limitation, no assumption about associations between exposure and outcome can be made [19]. Other researchers have performed case control studies based on hospital records [3,12]. No knowledge is available regarding the population-at-risk from which the cases arise in this design. Hence, they cannot be used for estimations of cancer incidence, although the odds ratio of cancer by breed can be estimated [20].

Due to the advanced stage of disease at time of diagnosis and the high frequency of metastasis, early detection is essential if treatment of canine gastric cancer is to be attempted. Because presenting signs may be similar to those observed in cases of chronic gastritis, some patients are symptomatically treated for gastritis for prolonged periods. The suspicion of gastric cancer may first arise when this form of treatment fails. It is therefore important for animal welfare to obtain a correct diagnosis as early as possible. Better knowledge of breeds at risk can enable early detection of gastric carcinoma in affected cases and may also facilitate

Table 1 Previous studies' results related to breed predisposition to gastric carcinoma in dogs

\begin{tabular}{|c|c|c|c|c|c|}
\hline Authors & Cases & Country & Breed predisposition & Study design & Comparison \\
\hline 1. (Lingeman et al. [9]) & 61 & USA & No breed predisposition & Case series & \\
\hline 2. (Sautter et al. [6]) & 14 & USA & No breed predisposition & Case series & \\
\hline 3. (Patnaik et al. [2]) & 26 & USA & No breed predisposition & Case series & \\
\hline 4. (Sullivan et al. [3]) & 31 & United Kingdom & $\begin{array}{l}7 / 31 \text { Rough collie and } 3 / 31 \\
\text { Staffordshire bull terrier }\end{array}$ & Case control & $\begin{array}{l}\text { Comparison with breeds of } \\
\text { the hospital accessions }\end{array}$ \\
\hline 5. (Fonda et al. [8]) & 15 & Italy & $\begin{array}{l}\text { Suggested genetic mechanism in } \\
8 \text { Belgian shepherd dogs } \\
\text { (7 Groenendael and } \mathbf{1} \text { Tervuren) }\end{array}$ & Case series & Pedigree examined in 8 cases \\
\hline 6. (Scanziani et al. [7]) & 11 & Italy & $\begin{array}{l}\text { High incidence in Belgian } \\
\text { shepherd dogs (Groenendael) }\end{array}$ & Case series & \\
\hline 7. (Penninck et al. [12]) & 16 & USA & 4/16 cases chow-chow (RR 46.2) & Case control & $\begin{array}{l}\text { Relative prevalence determined } \\
\text { against hospital population }\end{array}$ \\
\hline 8. (Swann and Holt. [5]) & 19 & USA & No breed predisposition & Case series & \\
\hline 9. (Bilek and Hirt. [11]) & 19 & Austria & 3/19 chow-chow (OR 23.5) & Case control & $\begin{array}{l}\text { Comparison with numbers from } \\
\text { the Austrian Kennel Club }\end{array}$ \\
\hline 10. (Qvigstad et al. [21]) & 4 & Norway & Norwegian Lundehund & Case series & \\
\hline 11. (Lubbes et al. [10]) & 92 & The Netherlands & Incidence: $1,18 \%$ among Tervuren & Retrospective cohort & \\
\hline
\end{tabular}


comparative genetic studies that could identify factors contributing to the development of gastric cancer.

We hypothesized that breed predisposition to canine gastric carcinoma exists in dogs. The aim of the study was to retrospectively investigate the proportion and possible breed predisposition to canine gastric carcinoma using data from the Norwegian Canine Cancer Register as a basis for calculating PMR for the period 1998-2009.

\section{Materials and methods \\ Study population}

Data were retrieved from the Norwegian Canine Cancer Register, a database consisting of all histopathologically verified tumours submitted to the diagnostic pathology laboratory at the Norwegian School of Veterinary Science between March 1998 and December 2009. The samples originated from private veterinary clinics all over Norway as well as from the Norwegian School of Veterinary Science. Information regarding each dog's name, breed, sex, age in years, and histological diagnosis is included in the database. The original referral forms were searched for information on methods of sampling, clinical signs, duration of signs, and nutritional status. The Norwegian Lundehund has been reported to be predisposed to gastric carcinoma [21], but gastrointestinal samples from Norwegian Lundehunds were not included in the registry for this time period due to a separate project involving this breed. The database was searched for the terms 'neoplasia' and 'stomach', and the combination of the two terms was defined as 'gastric cancer'.

\section{Histopathological examination}

Histopathological examination was performed on formalinfixed, paraffin-embedded tissue specimens stained with haematoxylin-eosin and van Gieson's. All histological slides classified as gastric cancer in the database were re-examined by an experienced veterinary pathologist (EJ) during 2011. Classification was performed according to the World Health Organization standard [4]. The pathologist was blind to the original histopathological diagnosis when reviewing the slides. Immunohistochemistry with cytokeratin and vimentin was performed if there was uncertainty regarding the diagnosis. The specimens diagnosed as carcinomas were included as cases in the study.

\section{Statistical analysis}

The number of gastric carcinomas in the registry was divided by the number of all other submitted tumours in order to yield the overall proportion of gastric carcinomas. The PMR of gastric carcinoma by breed was calculated by dividing the number of gastric carcinomas in a breed $\left(a_{1}\right)$ by all tumours in the breed $\left(n_{1}\right)$, over the number of gastric carcinomas in all other breeds $\left(a_{0}\right)$ divided by all other tumours in the other breeds in the database $\left(n_{0}\right)=\left(a_{1} / n_{1}\right) /$ $\left(a_{0} / n_{0}\right)$ [22]. The $95 \% \mathrm{CI}$ for the PMR was computed on a logarithmic scale [23]. The odds ratio (OR) for gastric carcinoma was calculated for males compared to females, and the P-value was estimated based on Fisher's exact test. The level of statistical significance was set at $\mathrm{P}<0.05$. All statistical analyses were performed using the software package Stata, Version 11 (Stata Corporation, College Station, Texas, USA).

\section{Results}

The material consisted of 19,715 tumours, of which 44 had been diagnosed as gastric cancer. In total, 10/44 (22.7\%) were given a new histopathological diagnosis when re-examined during 2011. 31/44 were confirmed as gastric carcinomas $(0.16 \%$ of total). The 13 cases which had been diagnosed with gastric cancer in the register, and which were not diagnosed as carcinomas, were found to be two carcinoma in situ, one adenoma, one hyperplasia, two metastasized tumours with unknown primary tumours, and six round cell tumours. In one case the diagnosis was inconclusive due to small and inadequate biopsies. Adenocarcinoma was the most prevalent carcinoma, occurring in $23 / 31$ (74\%) of the cases, whereas $8 / 31(26 \%)$ were undifferentiated carcinomas. In total, 14 of the 31 cases originated from the Norwegian School of Veterinary Science, and the remaining 17 cases originated from private clinics.

The sex of the affected dog was known for 19,489 tumours; the sex distribution is presented in Table 2. The OR for males versus females was $2.3(\mathrm{P}<0.02)$. The male to female ratio was 1.2:1. The age at diagnosis of a gastric tumour ranged from 1 year to 13 years, with a median age of 10 years. In 17 out of 31 cases the tumour had been sampled by gastroscopy. The remaining biopsies had been performed during exploratory laparotomies or during postmortems. The gastric location of the tumour was described for 19 of the cases and was restricted to the lesser curvature and/or pyloric region in 18 cases (95\%). In one case (5\%), the tumour was observed in the cardiac region.

Based on the referral sheets from the submitting veterinarian, clinical information was obtained for a subset of the included cases. The nutritional status was described in 16 cases, and characterized as either 'obese' $(1 / 16 ; 6 \%)$, 'good/ normal' $(5 / 16 ; 31 \%)$, or 'poor/thin' (10/16; 63\%). In 16 cases, the clinical signs were described. All of the dogs had

Table 2 Sex distribution among dogs with gastric carcinoma, recorded in the Norwegian canine cancer register (1998-2009)

\begin{tabular}{lccc}
\hline & Cases & Non-cases & Total \\
\hline Male & $17(0.25 \%)$ & $6,680(99.75 \%)$ & 6,697 \\
\hline Female & $14(0.11 \%)$ & $12,778(99.89 \%)$ & 12,792 \\
\hline & $31(0.16 \%)$ & $19,456(99.83 \%)$ & 19489 \\
\hline
\end{tabular}


experienced vomiting (16/16; 100\%), and haematemesis had occurred in only $2 / 16(13 \%)$ cases. Anorexia was observed in 6/16 (38\%) cases and weight loss in 5/16 (31\%) cases. Other, less frequently described signs were drooling, lethargy, diarrhoea, reduced general condition, abdominal pain, and polydipsia. The clinical signs are summarized in Table 3. The duration of signs prior to biopsy or euthanasia was usually described in terms of 'weeks' or 'months', but in 15 cases the duration was specified and ranged from 2 weeks to 6 months, with a median of 2 months.

Table 4 shows the total number of gastric carcinomas and PMR with $95 \%$ CI by breed. All samples from Tervuren $(n=7)$ and Groenendael $(n=3)$ breeds were diagnosed as adenocarcinomas. The value 1 represents the ratio for the average breed. Breeds in which the 95\% CI did not include 1 were considered to have significantly increased (or decreased) risk of gastric carcinoma. The breeds with significantly increased risk were found to be Tervuren, Bouvier des Flandres, Groenendael, collie (rough or smooth not specified), standard poodle, and Norwegian elkhound.

\section{Discussion}

In the present study, $0.16 \%$ of all canine tumours submitted to the diagnostic pathology laboratory at the Norwegian School of Veterinary Science during the period March 1998 to December 2009 were gastric carcinomas. This proportion corresponds to what Arnesen et al. (2001) found in their study of the Norwegian Canine Cancer Register from 1990-1998 [1].

Breed predisposition to gastric carcinoma was detected based on the PMR. The highest relative proportion of gastric carcinoma was observed in Tervuren, Bouvier des Flandres, Groenendael, collie (rough or smooth not specified), standard poodle, and Norwegian elkhound. Tervuren had a PMR of 56, indicating that this breed is 56 times more likely to be diagnosed with gastric

Table 3 Clinical signs in dogs with gastric carcinomas recorded in the Norwegian canine cancer register (1998-2009) $(n=16)$

\begin{tabular}{ll}
\hline Vomiting & 16 \\
\hline Anorexia & 6 \\
\hline Weight loss & 5 \\
\hline Haematemesis & 2 \\
\hline Drooling & 2 \\
\hline Lethargy & 2 \\
\hline Reduced general condition & 2 \\
\hline Melena & 1 \\
\hline Abdominal pain & 1 \\
\hline Polydipsia & 1 \\
\hline
\end{tabular}

Table 4 Cases of gastric carcinoma, tumours, and PMR by breed, recorded in the Norwegian canine cancer register (1998-2009) $(n=31)$

\begin{tabular}{lccccc}
\hline Breed & $\begin{array}{c}\text { Gastric } \\
\text { carcinomas }\end{array}$ & $\begin{array}{c}\text { All tumours } \\
\text { in the breed }\end{array}$ & $\begin{array}{c}\text { PMR } \\
\text { Tervuren }\end{array}$ & $\begin{array}{c}\mathbf{9 5} \text { Cl } \\
\text { low }\end{array}$ & $\begin{array}{c}\mathbf{9 5 \%} \text { Cl } \\
\text { high }\end{array}$ \\
\hline $\begin{array}{l}\text { Bouvier des } \\
\text { Flandres }\end{array}$ & 1 & 102 & 56.1 & 24.7 & 127.2 \\
Groenendael & 3 & 18 & 36.5 & 5.3 & 253.3 \\
Collie & 2 & 51 & 34.5 & 10.8 & 110.5 \\
Standard poodle & 3 & 273 & 7.6 & 2.3 & 25.0 \\
Norwegian & 2 & 219 & 6.1 & 1.5 & 25.6 \\
elkhound & & & & & \\
Nova Scotia duck & 1 & 118 & 5.5 & 0.8 & 40.3 \\
tolling retriever & & 124 & 5.3 & 0.7 & 38.3 \\
Leonberger & 1 & 331 & 2.0 & 0.3 & 14.3 \\
Border collie & 1 & 434 & 3.1 & 0.7 & 12.8 \\
Bichon fries & 2 & 534 & 2.5 & 0.6 & 10.4 \\
Dachshund & 2 & 544 & 2.4 & 0.6 & 10.2 \\
Gordon setter & 2 & 349 & 1.9 & 0.3 & 13.5 \\
Irish setter & 1 & 616 & 1.0 & 0.1 & 7.6 \\
Labrador retriever & 1 & 1194 & 0.5 & 0.1 & 3.8 \\
German shepherd & 1 & 1407 & 0.4 & 0.1 & 3.2 \\
English setter & 1 & & & &
\end{tabular}

carcinoma than the average breed in this database. Belgian shepherd dogs are bred as four separate types Tervuren, Groenendal, Mallanois, and Lakenois - but breeding between these types is permitted by the Norwegian Belgian shepherd dog club (NBFK) when specific conditions are fulfilled and with limited frequency. Registration of offspring is determined by the length, colour, and structure of the coat [24]. Even though the four types are closely related, there are no reports of increased risk of gastric carcinoma in the Mallanois or the Lakenois. It is unknown whether this is due to the Mallanois and Lakenois being less frequent types of Belgian shepherd dog or whether there is a different prevalence between the types.

Belgian shepherd dogs, both Groenendael and Tervuren types, and rough collies have previously been reported to be at increased risk of developing gastric carcinoma $[3,7,8,25]$. Single cases of gastric carcinoma have previously been reported in Norwegian elkhound, standard poodle, and Bouvier des Flandres [5,6,12], but the present study is the first time that these breeds have been found to be at risk of this type of cancer. In this study, the breed Bouvier des Flandres was found to have an increased risk of developing gastric carcinoma, but the wide 95\% CI for PMR renders the result uncertain, and it may not be clinically significant. 
It is worth noting that the breeds boxer, Rottweiler, and flat-coated retriever were not among the cases of gastric carcinoma in our study. These are all common breeds in Norway, and in previous studies based on the Norwegian Canine Cancer Register they were reported to have the highest relative risk ratio (RR) for developing tumours in general [1]. The database utilized for the current study included 616 tumour cases in boxers, 514 in Rottweilers, and 1,052 in flat-coated retrievers. A further interesting observation is the lack of mixed breeds among the cases of gastric carcinoma, although there are 1,932 tumours from mixed breeds in the database, making 'mixed' the most frequent 'breed'. Despite being the most common pure-bred dog in the database, with 1,407 tumours, the English setter is only represented with a single case. Chow chow and Staffordshire bull terrier breeds have previously been reported to have an increased risk of gastric carcinoma [3,11]. Both breeds were found to be infrequent in the database, with 14 tumour cases in chow chows and 22 tumour cases in Staffordshire bull terriers during the period 1998-2009. Neither of these breeds are represented in the current study material. This may be because these breeds are relatively uncommon in Norway, or that they have a different genetic composition compared to dogs from the same breeds in other countries.

Males were found to have significantly higher odds of gastric carcinoma than females in our study. This finding is in agreement with results from other studies and similar to what has been described in humans [2,3,6-10].

All dogs with gastric carcinoma in the current study, where clinical information was available, experienced vomiting. This, together with weight loss and anorexia, were the most commonly reported clinical signs. The complete clinical symptomatology may have been underreported, as a complete veterinary medical record was not available for all cases. In several cases, 'vomiting' was the only clinical sign reported, as this probably has been the main reason for diagnostic work-up, even though other clinical signs may have been present. The described clinical signs are in accordance with those reported in earlier studies $[3,5,6,8,9]$.

Dog breeds arise from a limited number of ancestors and combined with a frequent use of popular males, each domestic breed is an isolated population [26,27]. The genetic diversity within breeds is reduced, but there is a greater genetic divergence between breeds [26]. This makes the performance of genetic studies of dogs easier than those conducted in complex populations. Dogs share environments with their owners. Furthermore, because they naturally develop spontaneous cancers, in contrast to mouse models, they constitute an excellent comparative model for cancer [27]. The results from the study, identifying several breeds at risk, suggest that a genetic predisposition is of greater significance in dogs than humans, who have experienced a rapidly declining incidence of gastric carcinoma due to recent changes in the environment [16]. The dog may thus be a useful model for further comparative genetic studies of gastric cancer.

If differences in the ability of veterinarians to diagnose a gastric tumour and the willingness of dog owners to pursue and pay for diagnostic work-up are related to dog breed, they may influence the results of our study and be a source of selection bias. Underestimation can occur as a result of false negative biopsies. There might also be an underestimation of the prevalence of gastric cancer, as advanced disease and the age of an affected dog can result in owners being reluctant to pursue further diagnostic examinations.

A strength of the present study is the use of PMR to assess the proportion of all the affected animals in the population that have a particular disease under question. This allowed comparison with the background population of submitted cases, as opposed to the case series commonly reported in veterinary literature. However, a weakness of PMR is its sensitivity to variations in breed popularity and uncertainty in the estimates related to uncommon breeds. This is further influenced by the low prevalence of the disease. This might explain why some breeds previously described to have a higher risk are not represented in this study. It is also possible that a larger study would have been able to detect more breeds at increased risk among Norwegian dogs.

In the Norwegian Canine Cancer Register there is a higher proportion of females than males. This is probably due to mammary tumours dominating the register, as reported in previous studies [1]. Of the 44 cases that were diagnosed with gastric cancer in the Norwegian Canine Cancer Register, $22.7 \%$ were given a new histopathological diagnosis when they were re-evaluated in 2011. The proportion of cases given a new histopathological diagnosis corresponds to that found in previous studies based on the Norwegian Canine Cancer Register [28].

The breed predisposition observed in the current study indicates a genetic susceptibility to gastric carcinoma. This is in contrast to gastric cancer in humans, which in developed countries has declined significantly in incidence over the past 50 years, attributed largely to the eradication of $H$ pylori infection [16]. A reasonable interpretation is that environmental risk factors play a smaller role in the development of gastric cancer in dogs compared to humans.

\section{Conclusion}

The proportion of cases of gastric carcinoma recorded in the Norwegian Canine Cancer Register for the period March 1998 to December 2009 was found to be $0.16 \%$. 
A breed predisposition to gastric cancer was identified in the study, namely Tervuren, Bouvier des Flandres, Groenendael, collie, standard poodle, and Norwegian elkhound were found to have a significantly increased risk of gastric carcinoma according to the proportional morbidity ratios (PMRs).

\section{Abbreviations}

PMR: Proportional morbidity ratio; OR: Odds ratio; Cl: Confidence interval.

\section{Competing interest}

All authors declare that they have no competing interests.

\section{Authors' contribution}

TSW participated in the design of the study, preparing data from the database and performed the statistical analysis and drafted the manuscript EJ took part in designing the study and evaluated all the histological slides. AN took part in designing the study, helped with the statistical analysis and revised the manuscript. CB, TG and AMK participated in designing the study and revision of the manuscript. ES took part in designing the study, collected parts of the data and revised the manuscript. All authors read and approved the final version of the manuscript.

\section{Acknowledgements}

The authors thank the Norwegian Canine Cancer Register for access to their database, and Agria and the Swedish Kennel Club Research Fund for financial support.

\section{Author details}

${ }^{1}$ Department of Veterinary Clinical and Animal Sciences, Faculty of Health and Medical Sciences, University of Copenhagen, Frederiksberg, Copenhagen, Denmark. ${ }^{2}$ Department of Basic Sciences \& Aquatic Medicine, Norwegian School of Veterinary Science, Oslo, Norway. ${ }^{3}$ Department of Companion Animal Clinical Sciences, Norwegian School of Veterinary Science, Oslo, Norway. ${ }^{4}$ Cancer Registry of Norway, Oslo, Norway.

Received: 26 September 2012 Accepted: 4 March 2013

Published: 21 March 2013

\section{References}

1. Arnesen K, Gamlem H, Glattre E, Grøndalen J, Moe L, Nordstoga K: The Norwegian canine cancer register 1990-1998. Report from the project "Cancer in the dog". Eur J Comp Anim Pract 2001, 11:159-169.

2. Patnaik AK, Hurvitz Al, Johnson GF: Canine gastrointestinal neoplasms. Vet Pathol 1977, 14:547-555.

3. Sullivan M, Lee R, Fisher EW, Nash AS, Mccandlish IAP: A study of 31 cases of gastric carcinoma in dogs. Vet Rec 1987, 120:79-83.

4. Head KW: Tumours of lower alimentary tract. B World Health Organ 1976, 53:167-186.

5. Swann HM, Holt DE: Canine gastric adenocarcinoma and leiomyosarcoma: a retrospective study of 21 cases (1986-1999) and literature review. J Am Anim Hosp Assoc 2002, 38:157-164.

6. Sautter JH, Hanlon GF: Gastric neoplasms in the dog: a report of 20 cases. J Am Vet Med Assoc 1975, 166:691-696.

7. Scanziani E, Giusti AM, Gualtieri M, Fonda D: Gastric carcinoma in the Belgian shepherd dog. J Small Anim Pract 1991, 32:465-469.

8. Fonda D, Gualtieri M, Scanziani E: Gastric carcinoma in the dog - a clinicopathological study of 11 cases. J Small Anim Pract 1989, 30:353-360.

9. Lingeman CH, Garner FM, Taylor DON: Spontaneous gastric adenocarcinomas of dogs - review. J Natl Cancer Inst 1971, 47:137.

10. Wu HY, Rusiecki JA, Zhu KM, Potter J, Devesa SS: Stomach carcinoma incidence patterns in the United States by histologic type and anatomic site. Cancer Epidem Biomar 2009, 18:1945-1952.

11. Bilek A, Hirt RA: Breed-associated increased occurrence of gastric carcinoma in chow-chows. Wiener Tierarztl Monat 2007, 94:71-79.

12. Penninck DG, Moore AS, Gliatto J: Ultrasonography of canine gastric epithelial neoplasia. Vet Radiol Ultrasoun 1998, 39:342-348.

13. Wiinberg B, Spohr A, Dietz HH, Egelund T, Greiter-Wilke A, McDonough SP, Olsen J, Priestnall S, Chang YF, Simpson KW: Quantitative analysis of inflammatory and immune responses in dogs with gastritis and their relationship to Helicobacter spp. infection. J Vet Intern Med 2005, 19:4-14.

14. Couto CG, Rutgers HC, Sherding RG, Rojko J: Gastrointestinal lymphoma in 20 dogs. J Vet Intern Med 1989, 3:73-78.

15. KaserHotz B, Hauser B, Arnold P: Ultrasonographic findings in canine gastric neoplasia in 13 patients. Vet Radiol Ultrasoun 1996, 37:51-56.

16. Kelley JR, Duggan JM: Gastric cancer epidemiology and risk factors. J Clin Epidemiol 2003, 56:1-9.

17. Bornschein J, Malfertheiner P: Gastric carcinogenesis. Langenbecks Arch Surg 2011, 396:729-742.

18. Caldas C, Carneiro F, Lynch HT, Yokota J, Wiesner GL, Powell SM, Lewis FR, Huntsman DG, Pharoah PD, Jankowski JA, et al: Familial gastric cancer: overview and guidelines for management. J Med Genet 1999, 36:873-880.

19. Dohoo I, Martin W, Stryhn H: Introduction to observational studies. In Veterinary Epidemiologic Research. 2nd edition. Edited by McPike SM. Charlottetown, PEl, Canada: VER Inc; 2009:151-166.

20. Nodtvedt A, Berke O, Bonnett BN, Bronden L: Current status of canine cancer registration - report from an international workshop. Vet Comp Oncol 2011, 10:95-101.

21. Qvigstad G, Kolbjørnsen, Skancke E, Waldum HL: Gastric neuroendocrine carcinoma associated with atrophic gastritis in the Norwegian Lundehund. J Comp Pathol 2008, 139:194-201.

22. Dohoo I, Martin W, Stryhn H: Measures of disease frequency. In Veterinary Epidemiologic Research. 2nd edition. Edited by McPike SM. Charlottetown, PEl, Canada: VER Inc; 2009:73-90.

23. Dohoo I, Martin W, Stryhn H: Measures of association. In Veterinary Epidemologic Research. Edited by McPike SM. Charlottetown, PEl, Canada: VER Inc; 2009:135-150.

24. The Norwegian Belgian sheepdog club. 5-9-2012. http://www.nbfk.no/.

25. Lubbes D, Mandigers PJJ, Heuven HCM, Teske E: Incidence of gastric carcinoma in Dutch Tervueren shepherd dogs born between 1991 and 2002. Tijdschr Diergeneesk 2009, 134:606-610.

26. Shearin AL, Ostrander EA: Leading the way: canine models of genomics and disease. Dis Model Mech 2010, 3:27-34.

27. Rowell JL, McCarthy DO, Alvarez CE: Dog models of naturally occurring cancer. Trends Mol Med 2011, 17:380-388.

28. Nodtvedt A, Gamlem H, Gunnes G, Grotmol T, Indrebo A, Moe L: Breed differences in the proportional morbidity of testicular tumours and distribution of histopathologic types in a population-based canine cancer registry. Vet Comp Oncol 2011, 9:45-54.

doi:10.1186/1751-0147-55-25

Cite this article as: Seim-Wikse et al:: Breed predisposition to canine gastric carcinoma - a study based on the Norwegian canine cancer register. Acta Veterinaria Scandinavica 2013 55:25.

\section{Submit your next manuscript to BioMed Central and take full advantage of:}

- Convenient online submission

- Thorough peer review

- No space constraints or color figure charges

- Immediate publication on acceptance

- Inclusion in PubMed, CAS, Scopus and Google Scholar

- Research which is freely available for redistribution 